\title{
Atypical location of Becker's nevus: About two cases. Clinical and dermoscopic features
}

\section{Alaoui Aicha Elharrouni, Zakia Douhi, Samia Mrabat, Hanane Baybay, Sara Elloudi, Fatima Zahra Mernissi}

Department of Dermatology, University Hospital Hassan II Fez, Morocco

Corresponding author: Dr. Alaoui Aicha Elharrouni, E-mail: ealaouiaicha@gmail.com

\begin{abstract}
Becker's nevus $(\mathrm{BN})$ is a unilateral hyperpigmented, often hairy, cutaneous hamartoma, with geographic borders. It is usually localized on the shoulder, anterior chest, scapula, but there have been reports of BN in other areas (eg, lower extremities, face). In most cases, the lesion appears in late childhood or adolescence.we report 2 cases of Becker's Nevus with atypical location.
\end{abstract}

Key words: Becker's nevus; Atypical location; Dermoscopy

\section{INTRODUCTION}

The Becker nevus describes a frequently observed hamartomatous lesion characterized by circumscribed hyperpigmentation, often associated with an irregularly bordered hypertrichosis, is presented in unilateral localized fashion on upper trunk. Less common sites are extremities, face/neck, abdomen, and lower back, respectively. Due to an unusual presentation,we report tow cases of $\mathrm{BN}$ in atypical location.

\section{CASE REPORT}

The Becker nevus describes a frequently observed hamartomatous lesion characterized by circumscribed hyperpigmentation, often associated with an irregularly bordered hypertrichosis, is presented in unilateral localized fashion on upper trunk. Less common sites are extremities, face/neck, abdomen, and lower back, respectively. Due to an unusual presentation,we report tow cases og $\mathrm{BN}$ in atypical location.

\section{Case 1}

A 16-year-old male patient complained of a hyperpigmented lesion on cheek since the infant age.
She had no remarkable family history of disease. The patche was asymptomatic and became darker during puberty. Physical examination revealed an irregularborder hyperpigmented, and brownish macula on the cheek, with dark terminal hairs. The dermoscopy showed an Homogeneous diffuse pigmentation, Perifollicular hypopigmentation/follicles (Figs. la and lb). The clinical and dermoscopy aspect was compatible with Becker's nevus.

\section{Case 2}

A 40-year-old man visited our department, complaining of a pigmented skin lesion on the right upper arm and elbow. The patient had been in her usual state of good health, and had no remarkable abnormalities other than the skin lesion. The skin lesion had been there since birth. Skin examination revealed a well-defined, $10 \times 8 \mathrm{~cm}$ hairy pigmented patch with irregular borders and hypertrichosis in the right upper arm and elbow. Dermoscopic evaluation demonstrated well-defined pigment network, uniform thickness of lines, and uniform size and shape of network of pigmentation with perifollicular hypopigmentation (Fig. 2a and 2b). The histological study of a skin biopsy was in favor of a Becker's nevus.

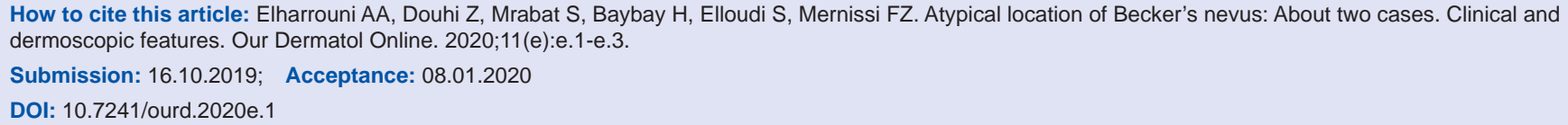




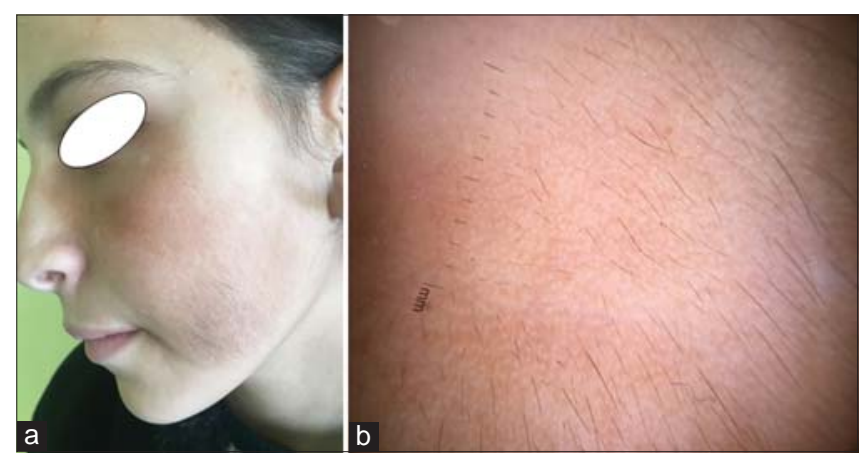

Figure 1: (a) A pigmented congenital skin lesion of $5 \times 6$ centimeters with irregular borders and hypertrichosis in the left cheek. (b) Dermoscopic aspect: showed an Homogeneous diffuse pigmentation, Perifollicular hypopigmentation/follicles.

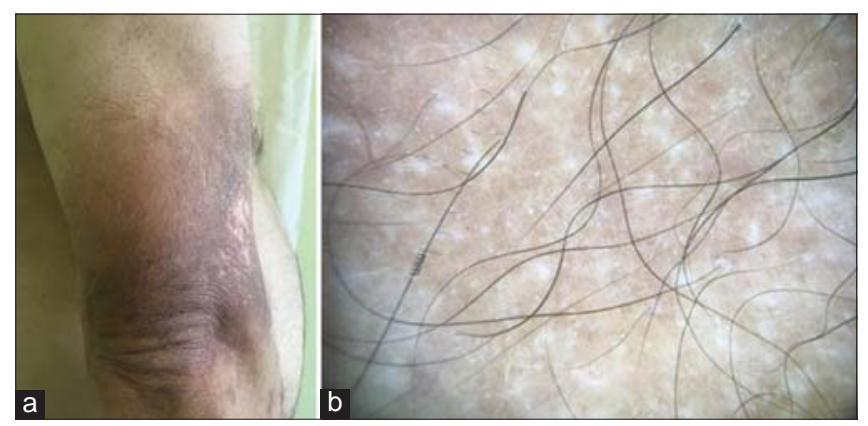

Figure 2: (a) A well-defined, $10 \times 8 \mathrm{~cm}$ hairy pigmented patch with irregular borders and hypertrichosis in the right upper arm and elbow. (b) Well-defined pigment network, uniform thickness of lines, and uniform size and shape of network of pigmentation with perifollicular hypopigmentation.

\section{DISCUSSION}

Becker nevus (BN) is a well-defined, irregular border, hyperpigmented cutaneous hamartoma, which is commonly associated with hypertrichosis and presented in unilateral localized fashion on upper trunk. However, it can occasionally occur as multiple or bilateral lesions on any parts of the body, less than $5 \%$ in areas considered unusual such as the, face, abdomen, upper arm and elbow, as the case in our patients. Becker's nevus, predominately seen in men, is an androgen-dependent lesion because it becomes more prominent after puberty [1]. Cilnicaly this nevus usually as a circumscribed, hyperpigmented patch with irregular outline that gradually enlarges with associated hypertrichosis, usually develops after the hyperpigmentation, and the hairs become coarser and darker with time [2]. Dermoscopy can substitute biopsy in cosmetically important areas. Although the diagnosis of Becker's nevus is primarily clinical, it can be aided by dermoscopy. In previous works of Vito Ingordo et al; network, focal, skin furrow and perifollicular hypopigmentation, hair follicles and vessels could be considered as peculiar dermoscopic features of BN [3]. Histological evaluation reveals acanthosis, epidermic hyperpigmentation, papillomatosis, arrector pili muscle hyperplasiam and melanophages in dermis $[4,5]$. Becker's nevus is a benign condition and there have been no reports of malignant transformation. Once established, it remains for the rest of one's life. No specific treatment has been described; since BN lesions are usually large, surgical treatment is limited. Treatment with Q-switched ruby and Erbium: YAG lasers have had satisfactory results. Also various successful depilation techniques can be used for cases with hypertrichosis [6,7].

\section{CONCLUSION}

To our knowledge, BN classically manifests unilaterally on the shoulder and upper trunk as a tan to brown patch or thin plaque. Our cases are presented to highlight the fact that Becker's nevus must be considered as a diagnostic possibility even in atypical sites even without hypertrichosis, if the history and clinical features are suggestive.

\section{Consent}

The examination of the patient was conducted according to the Declaration of Helsinki principles.

The authors certify that they have obtained all appropriate patient consent forms. In the form the patient(s) has/have given his/her/their consent for his/ her/their images and other clinical information to be reported in the journal. The patients understand that their names and initials will not be published and due efforts will be made to conceal their identity, but anonymity cannot be guaranteed.

\section{REFERENCES}

1. Hayashida MZ, Simões MM, Enokihara S, Hikawa RS, da Costa Pereira Cestar S. Acne arising on a facial Becker nevus following the lines of Blaschko. An Bras Dermatol. 2017;92:740-2.

2. Danarti R, Konig A, Salhi A, Bittar M, Happle R. Becker's nevus syndrome revisited. J Am Acad Dermatol. 2004;51:965-9.

3. Ingordo V Iannazzone SS, Cusano F, Naldi L. Dermoscopic features of congenital melanocytic nevus and becker nevus in an adult male population: an analysis with a 10 -fold magnifi cation. Dermatology. 2006;212:354-60.

4. Nair PA, Modasia K, Bhavsar N, Navadiya R. Dermatoscopy of Becker's nevus. Egyptian J Dermatol Venereol. 2009;39:37-9.

5. Pektas SD, Akoglu G, Metin A, Adiyaman NS, Demirseren ME. Becker nevus syndrome presentedwith ipsilateral breast hypoplasia. Indian J Dermatol. 2014;59:634.

6. Jung HJ, Moon SY, Sohn MY, Jang YH, Lee SJ, Kim DW, 
www.odermatol.com

et al. Some Becker's nevus melanocytes remain alive after treatment with q-switched alexandrite laser. Ann Dermatol. 2017; 29:352-5.

7. Lapidoth M, Adatto M, Cohen S, Ben-Amitai D, Halachmi S. Hypertrichosis in Becker's nevus: effective lowfluence laser hair removal. Lasers Med Sci. 2014;29:191-3.
Copyright by Alaoui Aicha Elharrouni, et al. This is an open access article distributed under the terms of the Creative Commons Attribution License, which permits unrestricted use, distribution, and reproduction in any medium, provided the original author and source are credited.

Source of Support: Nil, Conflict of Interest: None declared. 